\title{
Chapter
}

\section{THE ROLE OF POINT-OF-CARE TESTING IN THE DIAGNOSIS OF SYPHILIS}

Rita Castro $^{1^{*}}$ and Filomena Martins-Pereira ${ }^{2}$

${ }^{1}$ Global Health and Tropical Medicine (GHTM) Unidade de Microbiologia Médica/Instituto de Higiene e Medicina Tropical, Universidade Nova de Lisboa (UNL) Lisboa, Portugal ${ }^{2}$ Global Health and Tropical Medicine (GHTM) Unidade de Clínica Tropical / Instituto de Higiene e Medicina Tropical, Universidade Nova de Lisboa (UNL) Lisboa, Portugal 


\section{Contents}

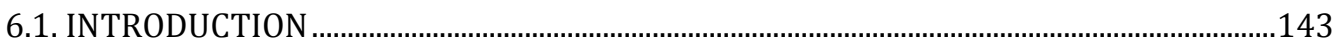

6.2. POINT-OF-CARE TESTING FOR SYPHILIS: RECENT ADVANCES ….................................146

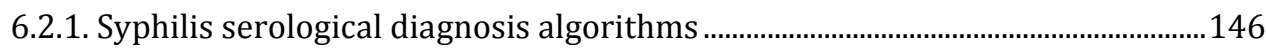

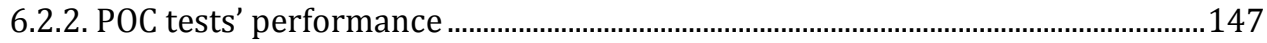

6.2.3. Impact of tests' introduction................................................................................ 148

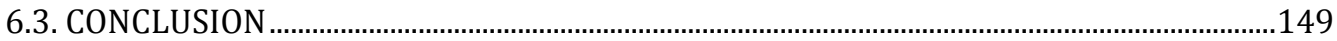

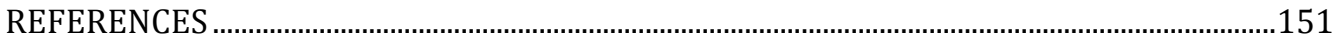




\subsection{INTRODUCTION}

Syphilis is a curable sexually transmitted disease caused by Treponema pallidum. Primary syphilis is generally characterised by a genital ulcer. Although often asymptomatic, it can still be transmitted to the sexual partners of infected persons. Globally, the World Health Organisation (WHO) estimates that 12 million cases of syphilis occur each year [1].

Infection during pregnancy is responsible for stillbirths, prematurity, and neonatal morbidity and mortality. Deaths by congenital syphilis affect more than one million babies a year in the world. Studies have also shown that syphilis has been associated with an increased risk of HIV transmission and acquisition [2,3]. Neurosyphilis may also develop earlier in the course of the disease in HIV-infected patients [4-8].

All these situations may be preventable if an early diagnosis and adequate treatment are performed, because treatment is inexpensive and there is no resistance to first-line treatment options. This is especially important with respect to congenital syphilis, since screening and treatment for syphilis during pregnancy are among the most cost-effective health interventions [9$11]$.

Laboratory diagnosis of syphilis is complex, since T.pallidum cannot be cultured, and serology is the main diagnostic methodology. When there is no laboratory testing available, the treatment of symptomatic syphilis (primary, secondary, and tertiary) can still be performed by taking into account the signs and symptoms of the different stages of the disease. However, diagnostic problems arise in asymptomatic or treated patients, who, in our experience, are the majority of cases [12-15].

Laboratory diagnosis is then necessary, which is based on both nontreponemal (the Rapid Plasma Reagin [RPR] or Venereal Disease Reference Laboratory [VDRL]) and treponemal (T. pallidum Hemagglutination Assay [TPHA], T. pallidum Particle Agglutination Assay [TPPA], or Fluorescence Treponemal Antibodies Absorption Test [FTA-ABS]) antibodies screening tests. Non-treponemal tests (NT) are simple to perform, inexpensive, and may be used to evaluate response to treatment, but whenever reactivity occurs, samples need to be confirmed with a treponemal test (TT). On the other hand, a reactive TT result cannot establish the difference between active and treated syphilis and cannot be used to evaluate treatment response [16-18].

These serological tests need trained personal and laboratory facilities, which are generally not available to screen outreach populations or in resourcelimited regions (mostly the parts of the world where syphilis is most prevalent), as well as in some other settings, such as screening clinics, where a rapid result is needed for immediate treatment. 
There is thus a need for rapid, inexpensive tests for the diagnosis of syphilis and other STIs that can be performed with no laboratory equipment by minimally trained staff and that involve few steps and easy interpretation. They should also give a result in a short period of time in view of patients' immediate treatment. These tests' characteristics have to fit in the so-called ASSURED Criteria (Table 1) [19-20], and they are known as point-of-care testing (POCT). A huge effort has been put into developing POCT in recent years, and as a consequence POCT for the diagnosis of HIV, hepatitis B and C, and other sexually transmitted infections (STIs), including syphilis, has been made available.

Table 1. Characteristics of an Ideal Rapid Test: ASSURED Criteria

$\mathbf{A}=$ Affordable (by all who need them)
$\mathbf{S}=$ Sensitive (minimum false negatives)
$\mathbf{S}=$ Specific (minimum false positives)
$\mathbf{U}=$ User-friendly (very simple to perform, minimal staff training, few steps)
$\mathbf{R}=$ Robust (not requiring refrigeration and storage) and rapid (results in less than
30 m to enable treatment at first visit)
$\mathbf{E}=$ Equipment-free (not needing complex laboratory equipment, may be performed
in whole, not requiring a centrifuge), easily collected non-invasive specimens
$\mathbf{D}=$ Deliverable to those who need them (not needing refrigeration and transport
conditions)

Adapted from Peeling et al. (2006) and Gaydos and Hardick (2014) [19,20]

Until recently, the POCT developed for syphilis included mostly TTs. Although NTs, such as the RPR, may be considered rapid tests, they are not POCT (Table 2), since they do not fulfil the characteristics pointed out in Table 1. Therefore, they cannot be used in places where laboratory facilities are not available.

One of the main problems with POCT is to make sure that its performance is not affected by conditions during transportation and in the places where needed, especially with respect to high temperatures and humidity. POCT requires temperatures between 4 and $30^{\circ} \mathrm{C}$. When temperatures are higher, it is important that a good quality control is performed on arrival of the tests and from time to time in a laboratory with basic conditions to perform other tests [21]. Studies on that subject are still needed.

The same should happen with respect to quality control and improvement. Studies on this subject [22-25] indicate that the quality of testing varies in different clinics and settings, but this can be overcome by rigorous quality assurance programmes for POCT. The identification of health care workers 
who may need further training can be done by external quality assurance (EQA) programmes.

Table 2. Characteristics of Rapid Non-Treponemal tests (RNTT) and PCTT Rapid Non-Treponemal tests (RNTT) and Point-of-Care Treponemal Tests (PCTT)

\begin{tabular}{cccc}
\hline & RNTT & POCNTT & POCTT \\
\hline Electricity & yes & no & no \\
Rotator & yes & no & no \\
Centrifuge & yes & no & no \\
Whole blood & no & yes & yes \\
Simple to perform & yes & yes & yes \\
Storage conditions $\left(\leq 30^{\circ} \mathrm{C}\right)$ & no & yes & yes \\
Transportation conditions $\left(\leq 30^{\circ} \mathrm{C}\right)$ & no & yes & yes \\
\hline Follow-up & yes & no & no \\
\hline Differentiation active vs past infection & yes & yes & no \\
\hline
\end{tabular}

The use of POCT will generally best benefit specific population groups, such as pregnant women (prenatal screening is affected by the lack of laboratory facilities, and therefore the availability of POCT will increase the number of pregnant women tested and decrease the number of infected foetuses) and atrisk populations, including sex workers and their clients, injection-drug users, and men who have sex with men (MSM) (decreases the number of infected adults in populations with the highest burden of syphilis, thus decreasing its transmission).

Screening pregnant women for syphilis is a universal recommendation. However, in Sub-Saharan Africa, where the burden of disease is highest, only around $40 \%$ of women attending antenatal clinics are screened. This is mainly because of the unavailability of laboratory conditions for classic syphilis tests to be performed in those clinics.

Screening this population group with POCT will also contribute to fulfilling one of the WHO objectives of the millennium, the elimination of congenital syphilis [26]. The choice of where to use POCT must also be considered. Generally, it should be used when patient access to syphilis screening is poor, especially among pregnant women, and in situations where its use will increase the number of people adequately and timely screened and treated. 


\subsection{POINT-OF-CARE TESTING FOR SYPHILIS: RECENT ADVANCES}

\subsubsection{Syphilis serological diagnosis algorithms}

Since the development of both NTs and TTs, they have been seen as complementing each other, and the rule was to perform the former, which is less expensive and has higher sensitivity but lower specificity than the latter. When reactive, confirmation with a specific test was mandatory. Using only one or the other of these tests leads to overtreatment, because we may be treating false positive reactions (only NTT reactive) and previously treated infections (only TT reactive). False positive reactions in persons without syphilis occur mainly in pregnancy (when the disease may be transmitted to the foetus), in some infections (which are more prevalent in places where the burden of disease is high), immunisations, injection-drug use, older age, and auto-immune conditions [18].

With the availability of point-of-care treponemal tests (POCTT), the possibility of TT automation, and the fact that some of these tests are less subjective, some authors recommend screening with a TT and confirming with an NTT $[17,27]$, which is called the reverse sequence algorithm. The European Centre for Disease Prevention and Control (ECDC) [28] and the British Association for Sexual Health and HIV (BASHH) [29] suggest a different approach, meaning that a TT should be used first, followed by a second TT when reactive. Since POCT includes mostly TTs, this recommendation could be adopted everywhere and become universal.

If only POCTT are available, all individuals whose samples show reactivity should be treated, even knowing that active and past infections cannot be differentiated with these types of tests. As a consequence, there is a possibility of overtreatment, and no treatment follow-up can take place.

Point-of-care non-treponemal tests (POCNTT) are nonetheless becoming available together with POCTT in so-called dual treponemal/non-treponemal POCT [30-33]. The possibility of performing both types of tests simultaneously makes the diagnosis of syphilis more specific, increasing positive predictive values and making it easier to differentiate between active and treated infection [34], thus decreasing the chances of inadequate treatment and overtreatment.

Since even dual POCNT and POCTT do not allow follow-up of response to treatment, they are less useful when laboratory facilities are available. On the contrary, they are of great diagnostic value in places where there are no conditions for a laboratory diagnosis. This is most important when screening pregnant women, whom it is better to overtreat than to undertreat [35].

The specific characteristics of POCNTT and POCTT are described in Table 3. Dual [25,36,39], triple [40-42], or quadruple [43] POCT for syphilis, HIV, and 
other STIs have recently been developed, and, for syphilis POCT alone, depending on the conditions and populations studied, quality control implementation and integration in local health facilities have shown to have good sensitivity and specificity for the tests included in the device.

\subsubsection{POC tests' performance}

Most POC tests for the diagnosis of syphilis have been evaluated in samples from pregnant women from developing countries (some in field conditions, others in central laboratories), although some studies have analysed samples from high-risk and other remote outreach group populations. In those studies, sensitivities of the POCTT varied from around $63 \%$ in the first studies to almost $100 \%$ [30-33,36,39,44,45], with specificities varying from around $90 \%$ to $100 \%$ in the most recent studies. The tests' increasing performance over the years is associated not only with the development of better tests but also with better quality control, one of the most important steps, especially when taking into consideration that these tests are to be used mainly in remote regions. In some of these studies, such as one in Delhi [45], POCTT still had good performances (sensitivity - $75 \%$, specificity - $100 \%$, positive predictive value - $100 \%$, negative predictive value $-99.45 \%$ ), even when in samples from lowprevalence $(2 \%)$ populations of pregnant women. The same was found by Malla et al. (2016) [46] in low-prevalence settings in Peru.

Lately, the performance of POCNTT, which are always dual tests with POCTT, has been evaluated. Yin et al. (2013) [32] analysed blood, finger-prick blood, and blood plasma samples with a dual test against reference standards. Sensitivity and specificity for the non-treponemal line varied from $86 \%$ to $88 \%$ and from $94 \%$ to $96 \%$, respectively. For the other line, variation for sensitivity and specificity was from $94 \%$ to $98 \%$ and $99 \%$ to $100 \%$, respectively. Castro et al. (2010) [30] found a reactive concordance in POCNTT of $98.4 \%$ and a non-reactive concordance of $98.6 \%$, whereas for POCTT they were $96.6 \%$ and $95.5 \%$, respectively. A sensitivity of $98.8 \%$ and a specificity of $94.7 \%$ for the POCNTT were also described by Castro et al. (2014), whereas the sensitivity and specificity of the treponemal POCTT was $99.5 \%, 98.9 \%$, $88.9 \%$, and $93.2 \%$, respectively, when compared with the T.pallidum hemagglutination assay and fluorescent treponemal antibody absorption tests [33]. Guinard et al. (2013) [31] found similar results, with the exception of a lower specificity for the non-treponemal line (76.6\%). Hess et al. [41], who described poor performance for syphilis of a triple POCT for HIV-HCV-syphilis, indicated that sensitivity improved when the gold standard was limited to samples from individuals more likely to be active cases.

A meta-analysis combining data from more than 7,200 patients on the performance of a combined treponemal and non-treponemal rapid diagnostic test for syphilis and yaws was undertaken by Marks etal. [47]. The test 
showed a good sensitivity, which was higher in patients with syphilis than in patients with yaws at low titres. The sensitivities of the treponemal and nontreponemal components for syphilis and yaws were $98.4 \%$ and $97.6 \%$ and $98.7 \%$ and $96.6 \%$, respectively, for high-titre specimens. The sensitivities for the POCTT and POCNT components were $93.1 \%$ for syphilis and $73.5 \%$ for yaws and $85.0 \%$ for syphilis compared to $59.1 \%$ for yaws. The specificity of the treponemal line was slightly higher in patients tested for syphilis when compared to yaws for low-titre specimens, with the inverse being true for the non-treponemal line. The overall agreement between the reference serology and the dual test was $85.2 \%$ (84.4-86.1\%), which is higher for past infection.

Attention should also be paid to the possibility of diagnosing neurosyphilis with POC tests. Ho et al. [48] have optimised three POC TTs for use in cerebrospinal fluid (CSF). One of the tests, the Syphicheck, was found to have a sensitivity of $62-64 \%$ and a specificity at or above a titre of $1: 4$ of $79-81 \%$, respectively. It was similar to the CSF-VDRL, which had a 54-69\% sensitivity and $73-75 \%$ specificity for laboratory and clinical neurosyphilis diagnoses, respectively. After neurosyphilis treatment, CSF-Syphicheck titres followed the same pattern as the CSF-VDRL.

\subsubsection{Impact of tests' introduction}

Studies on the impact of POC tests in screening pregnant women $[49,50]$ have reported that there was a substantial increase in antenatal syphilis screening following introduction of these types of tests. This was true for different settings: from urban to rural, primary health care centres or hospitals, and in countries with different health systems and cultural and socioeconomic contexts in Africa, Asia, and South America. The studies concluded that with this approach a larger number of women were screened and treated, with numbers that increased from non-available data to $96 \%$ (93.6\% treated), 51-95\% (97\% treated), 17.8-100\% (treated 91\%), 1.7-90.3\% (treated $103.6 \%$ ), and 79.9-95.6\% (treated 95.2\%) in China, Peru, Tanzania, Uganda, and Zambia, respectively.

In places where HIV screening was previously performed, this procedure was not affected. On the contrary, it is possible that HIV screening may have increased in some of those settings. Furthermore, Strasser et al. (2012) [51], Tucker et al. (2011) [52], and Smith et al. (2015) [42] showed that POC syphilis testing may be easily integrated into preexisting services, specifically for HIV. It should be noted that women not attending antenatal clinics (ANCs) will still continue to be missed and therefore not treated and neither will their partners.

When antenatal screening levels are already high, the introduction of POC tests may not be significant, even when well accepted by health professionals, as showed in a study performed in Mozambique. Actually, in some studies, the impact was more significant when testing was low at the baseline. In places 
where screening was high, the introduction of POC tests decreased coverage, probably due to changes in a well-organised process [53].

POCT seems to also be useful in outreach settings: in Canada [25], a resourcerich country, at-risk populations such as intravenous-drug users, sex workers, prisoners, and MSM seeking STI testing found the tests to be acceptable. New HIV and syphilis cases were then diagnosed and linked to treatment and care. The same was found by MacPherson et al. [54] in injecting-drug users (IDUs), MSM, and UK Africans, who successfully reached these target groups, many of whom would not have been tested otherwise. Studies performed in Bangalore, India, and the Amazon region of Brazil $[22,44,55,56]$ showed that POCT was helpful in alleviating the impact of syphilis in both pregnant and non-pregnant outreach populations, including high-risk individuals such as sex workers. Although this was also true in Guatemala [42], there screening uptake was limited by a shortage of health care workers and poor supply chain management.

POCT is generally well accepted by clients and health professionals to diagnose either HIV or syphilis, as demonstrated in Lima, Peru [57]; Manaus, Brazil [56]; India [40]; Kenya [58]; Tanzania [49,59]; China; Uganda; Zambia [49]; Canada [27]; and England [54]. Clients were satisfied in receiving the test results rapidly in the first visit $[24,49]$, with no need to come back for results. In some studies, clients were asked if they would prefer a finger prick to venipuncture; the answer was affirmative, especially because a smaller amount of blood was drawn [49]. Health workers also agreed that POC syphilis tests simplified screening procedures [59].

Health professionals and some authors also felt that training, supervision, and quality assurance must be strengthened and made an integrated part of POCT programmes $[24,50,60,61]$. Moreover, some health care workers stated that training would overcome their fear of communicating positive results and improve partners' notification [42].

The cost-effectiveness of POC tests should also be considered. Nowadays, antenatal screening and treatment of maternal syphilis are cost-effective health interventions [62], even under low prevalence of infection of 1 and $2 \%$, as shown by Kashiap et al. (2015) [45] and Mallma et al. (2015) [46], especially when using POCTT.

\subsection{CONCLUSION}

The difficulty in diagnosing syphilis, which is more common when asymptomatic, has been a problem for years, especially in places where laboratory diagnosis is not possible. Lately, it seems that this may be overcome with the availability of treponemal and non-treponemal POC tests that may be used successfully in areas with unfavourable conditions for a normal 
diagnostic laboratory. Overall, these tests have shown to have good sensitivities and specificities $[30,32,33,47]$ and to increase screening in different population groups from pregnant women to sex workers, MSM and drug addicts, and other outreach populations anywhere in the world $[22,25,44,54-56]$. The tests are generally well received by local health care workers and clients in places where studies have been performed $[24,25,40,49,56]$.

Although some years ago the cost-effectiveness of POCT was still a problem, nowadays treponemal rapid tests are shown to be cost-effective and seem to be a good screening option. However, using this type of test leads to overtreatment, and, as a consequence, there is concern about its use alone without a non-specific TT. In the case of pregnant women, the cheapest option would then be mass treatment, but adverse penicillin reactions, the impossibility of identifying sexual partners, difficulties in infrastructures to deliver treatment, and universal treatment acceptance by non-infected women make such a test difficult to implement [63]. Therefore, the new dual tests could overcome these problems in all types of populations, but they are still costly. When their price is reduced, which we hope will be in the near future, they will be the best option.

While dual tests with the previously described characteristics are not available and because the objective of POCT is to deliver adequate and immediate diagnosis and treatment to all those infected, no matter if they live in the most remote regions, POCTT remain the best option. They should be integrated with mother-to-child HIV prevention programmes, especially now that dual rapid syphilis/HIV tests are available, and included in countries' national guidelines. Ideally, they should be performed, when necessary, in conjunction with the new POC assays for diagnosis of other STIs, such as Chlamydia trachomatis, Neisseria gonorrhoeae, Trichomonas vaginalis, and herpes simplex virus, which are becoming more sensitive and specific and will eventually also become affordable. Nonetheless, screening and treatment will only be effective if intensive and continuous training, supervision, and quality assurance are strengthened wherever these programmes are used. 


\section{REFERENCES}

1. World Health Organization Sexually Transmitted Infections (Fact Sheet 110). Geneva: 2016. http://www.who.int/mediacentre/factsheets/fs110/en/ (accessed on the 2016-12-30).

2. J.N. Wasserheit. Sex. Transm. Dis. 19(2) (1992) 61-77.

3. K. Buchacz, P. Patel, M. Taylor, P.R. Kerndt, R.H. Byers, S.D. Holmberg, J.D. Klausner. AIDS 18(15) (2004) 2075-2079.

4. Centers for Disease Control and Prevention (CDC). MMWR Morb Mortal Wkly Rep 56(25) (2007) 625-628.

5. B. Stoner. Clin. Infect. Dis. 44(S3) (2007) S130-S146.

6. N.M. Zetola, J.D. Klausner. Clin. Infect. Dis. 44(9) (2007) 1222-1228.

7. E.L. Ho, S.S. Spudich. Sex. Health 12(2) (2015) 148-154.

8. E. Firlag-Burkacka, P. Swiecki, I. Cielniak, E. Siwak, J. Gizinska, E. Bakowska, J. Kubicka, P. Pulik, J. Kowalski, R. Podlasin, A. Horban. HIV Med. 17(5) (2016) 323-326.

9. F Terris-Prestholt, D. Watson-Jones, K. Mugeye, L. Kumaranayake, L. Ndeki, H. Weiss, J. Changalucha, J. Todd, F. Lisekie, B. Gumodoka, D. Mabey, R. Hayes. Sex. Transm. Infect. 79(5) (2003) 375-381.

10. X.S. Chen, R.W. Peeling, Y.P. Yin, D. Mabey. Future Microbiol. 6(10) (2011) 1131-1134.

11. S. Hawkes, N. Matin, N. Broutet, N. Low. Lancet Infect. Dis. 11(9) (2011) 684-691.

12. R.R. Castro, E.S. Prieto, I. Santo, J. Azevedo, F.L. Exposto. Am. J. Clin. Pathol. 116(4) (2001) 581-585.

13. R. Castro, E.S. Prieto, I. Santo, J. Azevedo, L. Exposto Fda. J. Clin. Microbiol. 41(1) (2003) 250-253.

14. R. Castro, E. Prieto, M.J. Águas, M.J. Manata, J. Botas, I. Santo, J. Azevedo, F.L. Pereira. Int. J. STD AIDS 18(12) (2007) 842-825.

15. R. Castro, E. Prieto, M.J. Águas, M.J. Manata, J. Botas, F.M. Pereira. J. Clin. Microbiol. 47(8) (2009) 2510-2512.

16. S.A. Larsen, B.M. Steiner, A.H. Rudolph. Clin. Microbiol. Rev. 8(1) (1995) 1-21.

17. R. Ballard, E.W. Hook III. Laboratory diagnosis of sexually transmitted infections, including human immunodeficiency virus. World Health Organization (WHO), Geneva, Switzerland, 2013, p. 107-129.

18. T.R. Frieden, H.W. Jaffe, J. Cono, C.L. Richards, M.F. Iademarco MF. Sexually Transmitted Diseases Treatment Guidelines, 2015, MMWR Recomm. Rep. 2015; 64 (No. RR-3), p. 34-49.

19. R.W. Peeling, K.K. Holmes, D. Mabey, A. Ronald. Sex. Transm. Infect. 82(5) (2006) 1-6.

20. C. Gaydos, J. Hardick. Exp. Rev. Anti. Infect. Ther. 12(6) (2014) 657-672.

21. World Health Organization. The use of Rapid Syphilis Tests. Special Programme for Research \& Training in Tropical Diseases (TDR) sponsored by UNICEF/UNDP/World Bank/WHO. 2006 WHO/TDR/SDI/06.1 http://apps.who.int/iris/bitstream/10665/43590/1/TDR SDI 06.1 eng.pdf

22. S. Mishra, B. Naik, B. Venugopal, P. Kudur, R. Washington, M. Becker, J. Kenneth, K. Jayanna, B.M. Ramesh, S. Isac S, M.C. Boily, J.F. Blanchard, S. Moses. Sex. Transm. Infect. 86(3) (2010) 193-198. 
23. L. Severe, D. Benoit, X.K. Zhou, J.W. Pape, R.W. Peeling, D.W. Fitzgerald, K.S. Mate. J. Sex. Transm. Dis. (2013) 247901.

24. P.W. Smit, D. Mabey, T. van der Vlis, H. Korporaal, J. Mngara, J. Changalucha, J. Todd, R.W. Peeling. BMC Infect. Dis. 13 (2013) 530.

25. J. Bergman, J. Gratrix, S. Plitt, J. Fenton, C. Archibald, T. Wong, A.E. Singh. AIDS Res. Treat. (2013) 819593.

26. World Health Organization. The Global elimination of congenital syphilis: rationale and strategy for action. World Health Organization (WHO), Geneva, Switzerland, 2007.

http://apps.who.int/iris/bitstream/10665/43782/1/9789241595858 eng.pd f.

27. P.N. Levett, K. Fonseca, R.S. Tsang, K. Kadkhoda, B. Serhir, S.M. Radons, M. Morshed. Can. J. Infect. Dis. Med. Microbiol. 26(S. A9) (2015) 6A-12A.

28. M Janier, V. Hegyi V, Dupin N, M. Unemo, G.S. Tiplica, M. Potočnik, P. French, R. Patel. J. Eur Acad. Dermatol. Venereol. 28(12) (2014) 1581-1593.

29. M. Kingston, P. French, S. Higgins, O. McQuillan, A. Sukthankar, C. Stott, B. McBrien, C. Tipple, A. Turner, A.K. Sullivan; Members of the Syphilis guidelines revision group 2015, K. Radcliffe, D. Cousins, M. FitzGerald, M. Fisher, D. Grover, S. Higgins, M. Kingston, M. Rayment, A. Sullivan. Int. J. STD AIDS 27(6) (2016) 421-246.

30. A.R. Castro, J. Esfandiari, S. Kumar, M. Ashton, S.E. Kikkert, M.M. Park, R.C. Ballard. J. Clin. Microbiol. 48(12) (2010) 4615-4619.

31. J. Guinard, T. Prazuck, H. Péré, C. Poirier, J. LeGoff, E. Boedec, A. Guigon, N. Day, L. Bélec. Int. J. STD AIDS 24(12) (2013) 944-950.

32. Y.P. Yin, X.S. Chen, W.H. Wei, K.L. Gong, W.L. Cao, G. Yong, L. Feng, S.J. Huang, D.M. Wang, Y. Han, S.C. Chen, D. Mabey, R.W. Peeling. Clin. Infect. Dis. 56(5) (2013) 659-665.

33. R. Castro, Â Lopes, F.L. Martins Pereira. Sex. Transm. Dis. 41(8) (2014) 467-469.

34. L.M. Causer, J.M. Kaldor, D.P. Conway, D.E. Leslie, I. Denham, T. Karapanagiotidis, C. Ryan, H. Wand, D.A. Anderson, P.W. Robertson, A.M. McNulty, B. Donovan, C.K. Fairley, R.J. Guy. Clin. Infect. Dis. 61(2) (2015) 184-191.

35. S. Tuddenham, K.G. Ghanem. BMC Infect. Dis. 19 (2015) 15:351.

36. Y.P. Yin, E. Ngige, C. Anyaike, G. Ijaodola, T.A. Oyelade, R.G. Vaz, L.M.Newman, X.S. Chen. Int. J. Gynaecol. Obstet. 130(S1) (2015) S22-S26.

37. S.R. Leon, L.B. Ramos, S.K. Vargas, N. Kojima, D.G. Perez, C.F. Caceres, J.D. Klausner. J. Clin. Microbiol. 54(2) (2016) 492-494.

38. C.C. Bristow, S.L. Leon, E. Huang, B.J. Brown, L.B. Ramos, S.K. Vargas, J.A. Flores, C.F. Caceres, J.D. Klausner. Sex. Transm. Infect. 92(3) (2016) 182-185.

39. C.C. Bristow, L. Severe, J.W.Pape, M. Javanbakht, S.J. Lee, W.S. Comulada, J.D. Klausner. BMC Infect. Dis. 16 (2016) 302.

40. N.P. Pai, J. Kurji, A. Singam, R. Barick, Y. Jafar, M.B. Klein, S. Chhabra, P. Shivkumar. Int. J. STD AIDS. 23(5) (2012) 319-324.

41. K.L. Hess, D.G. Fisher, G.L. Reynolds. PLoS One. 9(11) (2014) 112190.

42. A. Smith, M. Sabidó, E. Camey, A. Batres, J. Casabona. Int. J. Gynaecol. Obstet. 130(S1) (2015) S70-72.

43. N.P. Pai, R. Dhurat, M. Potter, T. Behlim, G. Landry, C. Vadnais, C. Rodrigues, L. Joseph, A. Shetty. BMJ Open 4(12) (2014) e005040. 
44. A.S. Benzaken, M. Sabidó, E. Galban E, Pedroza V,A.J. Araújo, R.W. Peeling, D. Mabey. Int. J. STD AIDS 22(1) (2011) 15-18.

45. B. Kashyap, T. Sagar, I.R. Kaur. Indian J. Sex. Transm. Dis. 36(2) (2015) 162165.

46. P. Mallma, P. Garcia, C. Carcamo, S. Torres-Rueda, R. Peeling, D. Mabey, F Terris-Prestholt. PLoS One. 11(3) (2016) e0149568.

47. M. Marks, Y.P. Yin, X.S. Chen, A. Castro, L. Causer, R. Guy, R. Wangnapi, O. Mitjà, A. Aziz, R. Castro, F.L. Martins Pereira, F. Taleo, J. Guinard, L. Bélec, Y. Tun, C. Bottomley, R.C. Ballard, D.C. Mabey. Clin. Infect. Dis. 63(5) (2016) 627-633.

48. E.L. Ho, L.C. Tantalo, T. Jones, S.K. Sahi, C.M. Marra. Sex Transm. Dis. 42(1) (2015) 48-52.

49. D.C. Mabey, K.A. Sollis, H.A. Kelly, A.S. Benzaken, E. Bitarakwate, J. Changalucha, X.S. Chen, Y.P. Yin, P.J. Garcia, S. Strasser, N. Chintu, T. Pang, F. Terris-Prestholt, S. Sweeney, R.W. Peeling. PLoS Med. 9(6) (2012) e1001233.

50. É.M. Ansbro, M.M. Gill, J. Reynolds, K.D. Shelley, S. Strasser, T. Sripipatana, A. Tshaka Ncube, G. Tembo Mumba, F. Terris-Prestholt, R.W. Peeling, D. Mabey. PLoS One. 10(6) (2015) e0127728.

51. S. Strasser, E. Bitarakwate, M. Gill, H.J. Hoffman, O. Musana, A. Phiri, K.D. Shelley, T. Sripipatana, A.T. Ncube, N.J. Chintu. J. Acquir. Immune Defic. Syndr. 61(3) (2012) e40-e46.

52. J.D. Tucker, L-G. Yang, B. Yang, H.P. Zheng, H. Chang, C. Wang, S.Y. Shen, Z.J. Zhu, Y.P. Yin, S.V. Subramanian, X.S. Chen, M.S. Cohen. J. Acquir. Immune Defic. Syndr. 57(5) (2011) e106-e111.

53. C. De Schacht, C. Lucas, N. Sitoe, R. Machekano, P. Chongo, M. Temmerman, 0. Tobaiwa, L. Guay, S. Kassaye, I.V. Jani. PLoS One. 10(8) (2015) e0135744.

54. P. MacPherson, A. Chawla, K. Jones, E. Coffey, V. Spaine, I. Harrison, P. Jelliman, P. Phillips-Howard, C. Beynon, M. Taegtmeyer. BMC Public Health 11(419) (2011).

55. L.V. Ribeiro, M. Sabidó, E. Galbán, J.A. Guerra, D. Mabey, R.W. Peeling, A.S. Benzaken. Sex. Transm. Infect. 91(2) (2015) 94-96.

56. M. Sabidó, A.S. Benzaken, E.J. de-Andrade-Rodrigues, P. Mayaud. Emerg. Infect. Dis. 15(4) (2009) 647-649.

57. E.C. Flores, M.E. Lluque, M. Chiappe, R. Lino, A.M. Bayer. Int. J. STD AIDS 26(10) (2015) 723-728.

58. E. Fleming, J. Oremo, K. O'Connor, A. Odhiambo, T. Ye, S. Oswago, C. Zeh, R. Quick, M.L. Kamb. J. Sex Transm. Dis. (2013) 674584.

59. S. Nnko, J. Changalucha, J. Mosha, C. Bunga, J. Wamoyi, R. Peeling, D. Mabey. Health Policy Plan. 31(5) (2016) 667-673.

60. A.S. Benzaken, M.L. Bazzo, E. Galban, I.C. Pinto, C.L. Nogueira, L. Golfetto, N.S. Benzaken, K.A. Sollis, D. Mabey, R.W. Peeling. Sex Transm. Infect. 90(1) (2014) 14-18.

61. C.C. Bristow, E. Larso, M. Javanbakht, E. Huang, L. Causer, J.D. Klausner. Sex Health 12(2) (2015) 119-125.

62. B.R. Schackman, C.P. Neukermans, S.N. Fontain, C. Nolte, P. Joseph, J.W. Pape, D.W. Fitzgerald. PLoS Med. 4(5) (2007) e183.

63. F. Terris-Prestholt, P. Vickerman, S.Torres-Rueda, N. Santesso, S. Sweeney, P. Mallma, K.D. Shelley, P.J. Garcia, R. Bronzan, M.M. Gill, N. Broutet, T. Wi, C. Watts, D. Mabey, R.W. Peeling, L. Newman. Int. J. Gynaecol. Obstet. 130(1) (2015) S73-S80. 
